\title{
Centrally Mediated Disorders of Gastrointestinal Pain
}

\author{
Laurie Keefer, ${ }^{1}$ Douglas A. Drossman, ${ }^{2}$ Elspeth Guthrie, ${ }^{3}$ Magnus Simrén, ${ }^{4}$ \\ Kirsten Tillisch, ${ }^{5}$ Kevin Olden, ${ }^{6}$ and Peter J. Whorwell ${ }^{7}$
}

${ }^{1}$ Division of Gastroenterology, Icahn School of Medicine at Mount Sinai, New York, New York; ${ }^{2}$ Center for Functional Gl and Motility Disorders, University of North Carolina and Center for Education and Practice of Biopsychosocial Care LLC, Drossman Gastroenterology PLLC, Chapel Hill, North Carolina; ${ }^{3}$ Mental Health and Social Care Trust, Manchester Royal Infirmary, Manchester, UK; ${ }^{4}$ Department of Internal Medicine and Clinical Nutrition, Institute of Medicine Sahlgrenska Academy, University of Gothenburg, Gothenburg, Sweden; ${ }^{5}$ Oppenheimer Family Center for Neurobiology of Stress Division of Digestive Diseases, David Geffen School of Medicine at UCLA, Los Angeles, California; ${ }^{6}$ SJHMC Internal Medicine Department, Phoenix, Arizona; and ${ }^{7}$ Education and Research Centre Wythenshawe Hospital, Manchester, UK

Centrally mediated abdominal pain syndrome, formerly known as functional abdominal pain syndrome, can be distinguished from other functional gastrointestinal disorders by its strong central component and relative independence from motility disturbances. Centrally mediated abdominal pain syndrome is a result of central sensitization with disinhibition of pain signals rather than increased peripheral afferent excitability. A newly described condition, narcotic bowel syndrome/opioidinduced gastrointestinal hyperalgesia, is characterized by the paradoxical development of, or increases in, abdominal pain associated with continuous or increasing dosages of opioids. Patients only have relief when opioids are withdrawn. We define both conditions in the context of epidemiology, pathophysiology, clinical evaluation, and treatment, emphasizing the importance of a physicianpatient relationship in all aspects of care.

Keywords: Chronic Abdominal Pain; Narcotic Bowel; Functional Abdominal Pain; Centrally Mediated Pain; Rome IV.

$\mathrm{T}$ his paper describes our approach and recommendations related to 2 gastrointestinal (GI) disorders whose primary symptoms are believed to have a central determinant-centrally mediated abdominal pain syndrome (CAPS), formerly known as functional abdominal pain syndrome, and a new condition, narcotic bowel syndrome (NBS)/opioid-induced GI hyperalgesia.

\section{D1. Centrally Mediated Abdominal Pain Syndrome}

\section{Definition}

CAPS is characterized by continuous, nearly continuous, or frequently recurrent abdominal pain that is often severe and only rarely related to gut function. CAPS is associated with loss of function across several life domains, including work, intimacy, social/leisure, family life, and caregiving for self or others, and must be present for at least 6 months before diagnosis.

Like other functional gastrointestinal disorders (FGID), CAPS cannot be explained by a structural or metabolic disorder using currently available diagnostic methods. Abdominal pain can be produced by or attributed to nondigestive organs, such as those in the urinary or gynecologic systems, and disorders in these locations that explain such pain should be excluded before the diagnosis of CAPS can be established. A substantial proportion of CAPS patients suffer significant negative contributions from multiple, probably unnecessary, surgical interventions performed in an attempt to address their pain complaints, ${ }^{1}$ and attribute their pain to "adhesions." Adhesions can cause symptoms of acute or subacute obstruction, which in turn cause pain, but there is no good evidence that adhesions themselves are a cause for chronic unrelenting pain, such as that seen in CAPS. ${ }^{2}$

The predominance of pain as the central complaint, almost to the exclusion of other symptoms, distinguishes CAPS from other painful FGID, such as irritable bowel syndrome (IBS) and functional dyspepsia (FD), primarily by the poor relationship of pain with food intake or defecation. CAPS may represent the far end of the spectrum of IBS severity, where psychosocial factors and more generalized central hypersensitivity predominate. It is distinguished from chronic pelvic pain by its abdominal location and from "abdominal migraine" in that the pain from CAPS is constant rather than cyclical.

Pain associated with CAPS may be colicky in nature, as in IBS, although it tends to be more prolonged and widespread. Another description that is quite common, especially after a previous surgery, is that pain is burning in character; this form is particularly challenging to treat. ${ }^{3}$ CAPS can be associated with other unpleasant somatic symptoms and syndromes, such as fibromyalgia and chronic fatigue syndrome. While not part of the diagnostic criteria, psychological comorbidities are common when pain is persistent

Abbreviations used in this paper: CAPS, centrally mediated abdomina pain syndrome; FD, functional dyspepsia; FGID, functional gastrointestinal disorder; GI, gastrointestinal; IBS, irritable bowel syndrome; NBS, narcotic bowel syndrome; SNRI, serotonin-norepinephrine reuptake inhibitor; SSRI, selective serotonin reuptake inhibitor; TCA, tricyclic antidepressant; TLR, Toll-like receptor.

(Q) Most current article

(C) 2016 by the AGA Institute $0016-5085 / \$ 36.00$

http://dx.doi.org/10.1053/j.gastro.2016.02.034 
over a long period of time, are further associated with chronic pain behaviors, and dominate the patient's life. ${ }^{3}$

\section{Epidemiology}

CAPS is considered less common than other FGIDs, such as functional heartburn, FD, or IBS, with prevalence data ranging from $0.5 \%$ to $2.1 \%{ }^{4}$ CAPS seems to be between 1.5 and 2 times more common in women, 4,5 and its prevalence reaches a peak in the fourth decade of life (35-44 years in the US householder survey) and then decreases with age. ${ }^{6}$

Approximately $80 \%$ of CAPS patients have consulted a physician, and half had seen a physician between 1 and 3 times per year specifically for abdominal pain, ${ }^{4,7} 4$ times more frequently than people without abdominal complaints. CAPS patients in the United Kingdom required 5.7 consultant visits, completed 6.4 endoscopic or imaging investigations, and underwent 2.7 surgical interventions (primarily hysterectomy and exploratory laparotomy) during a follow-up period of 7 years. ${ }^{8}$ In the United States, CAPS patients missed work a mean of 11.8 days in the previous year, 3 times more than subjects without abdominal symptoms, and "felt too sick to go to work" at the moment of the survey in $11.2 \%$ of cases, about 3 times more frequently than respondents without FGIDs. ${ }^{4}$

D1. Diagnostic Criteria $^{a}$ for Centrally Mediated Abdominal Pain Syndrome ${ }^{b}$

Must include all of the following:

- Continuous or nearly continuous abdominal pain

- No or only occasional relationship of pain with physiological events (eg, eating, defecation, or menses) ${ }^{c}$

- Pain limits some aspect of daily functioning ${ }^{d}$

- The pain is not feigned

- Pain is not explained by another structural or functional gastrointestinal disorder or other medical condition

${ }^{a}$ Criteria fulfilled for the last 3 months with symptom onset at least 6 months before diagnosis.

${ }^{b}$ CAPS is typically associated with psychiatric comorbidity, but there is no specific profile that can be used for diagnosis.

${ }^{c}$ Some degree of gastrointestinal dysfunction may be present.

${ }^{d}$ Daily function could include impairments in work, intimacy, social/leisure, family life, and caregiving for self or others.

\section{Pathophysiology}

The biology of CAPS is likely similar to other chronic visceral pain disorders, such as IBS, FD, and interstitial cystitis. While these disorders are all defined by discrete symptom criteria, they have in common comorbidity with other pain syndromes, predisposing life events, and treatment responses. As with many chronic somatic pain disorders, CAPS does not fit easily into the traditional categories of neuropathic or inflammatory pain. Rather, alterations in modulatory and motivational pain dimensions play a major role in both the generation and perpetuation of CAPS.

Altered central sensory processing in gastrointestinal pain syndromes: lessons learned from irritable bowel syndrome and functional dyspepsia. The brain receives interoceptive input from the abdominal viscera, which is then combined with cognitive, emotional, and other sensory information for conscious interpretation in the anterior insula. Neuroimaging studies in IBS are consistent with an abnormality in central processing of pain signals, with functional and structural abnormalities noted in sensory (mid-cingulate, insular, and somatosensory cortices, and thalamus), emotional arousal (anterior cingulate cortex, amygdala), and prefrontal cortical modulatory regions. Modulation of descending pain regulatory pathways in the brainstem by these cortical regions can lead to exaggerated sensitivity to both noxious and innocuous stimuli. Evidence that patterns of brain activation during anticipation of experimental pain are abnormal in IBS further supports this pathophysiologic model. Patients with FD show similar abnormalities compared with healthy control subjects. $^{9}$

One way in which CAPS differs from IBS and FD is that the pain symptoms are, by definition, reported as more constant and unrelated to peripheral events, such as food intake or defecation. This suggests that, unlike IBS and FD, the phasic, physiologic visceral afferent input from the gut plays a lesser role in symptom generation. These observations, along with the common responsiveness of CAPS symptoms to low-dose tricyclic antidepressants (TCA), raises the question of whether some CAPS patients have a peripheral or gut-based neuropathic pathophysiologic process. Unfortunately, neither the characteristically enlarged pain referral areas nor the response to TCAs (which work on both peripheral and central neuropathic pain conditions) make it possible to differentiate between these possibilities. However, even in the setting of a peripheral insult, once central sensitization is established, symptoms can persist in the absence of ongoing abnormal peripheral stimulation or worsen with minimal stimulation. ${ }^{10}$ Because no consistent initiating triggers are noted in CAPS, and the risk factors seem to be primarily psychosocial, it is presumed that central processes, such as altered descending pain modulation, are responsible for the chronicity of CAPS. ${ }^{11}$

Altered brain structure in chronic pain. Altered brain structure has also been described in multiple visceral and somatic pain disorders. In women with IBS, increased cortical thickness in the somatosensory cortex and decreased cortical thickness in regions of pain processing, including the insula and anterior cingulate cortex, is observed. ${ }^{12}$ IBS symptom severity was negatively correlated with the cingulate thickness, suggesting a role for loss of neural density in symptom generation. Using another metric 
of brain structure, gray matter volume, IBS patients had decreased volumes in widespread regions, including the insula, amygdala, cingulate, and brainstem, with early life trauma playing a role in these differences. ${ }^{13}$ Patients with FD also exhibit altered brain structure, with decreased gray matter density in multiple brain regions, including the insula and prefrontal cortex. ${ }^{14}$ Both IBS and FD have been shown to have abnormal structure of the brain's white matter tracts, with similar areas involved in the processing of pain and emotion. The role of structural change in functional pain disorders is not clear, with some debate as to whether these changes are pre-existing vulnerability factors for chronic pain, or side effects of the pain itself. Given the severity and chronicity of pain symptoms in patients with CAPS, the likelihood that structural brain changes exist is high.

Genetic and environmental vulnerability to centrally mediated abdominal pain syndrome. Animal models and human studies suggest that complex genetic influences play an important role in the predisposition to chronic pain. It is considered likely that this predisposition is a combination of genetic, environmental, and behavioral factors. Early evidence suggests genes related to serotonin reuptake, mucosal barrier function, pro- and antiinflammatory cytokines, among others, may be involved. Clinical and preclinical evidence suggests that there is a strong association of aversive early life events and certain types of psychosocial stressors with increased pain reports and changes in brain function among patients with FGID. ${ }^{15,16}$ The combination of genetic factors, learned behavioral factors, adverse early life events, and adult stress might determine, in part, the effectiveness of endogenous pain modulation systems and thereby influence development of CAPS.

Psychological factors can amplify the experience of pain, lending further rationale for the use of psychological interventions for the management of CAPS. For example, depression and anxiety mediate the effect of pain on function in chronic low back pain ${ }^{17}$ and a trauma history can negatively influence pain experience, coping, and the doctor-patient relationship. ${ }^{18}$ Finally, there is strong empirical support for the importance of pain catastrophizing, $^{19}$ fear avoidance behavior, ${ }^{20}$ self-efficacy, ${ }^{21}$ lack of perceived control, ${ }^{22}$ and passive pain coping ${ }^{23}$ on pain experience.

\section{Clinical Evaluation}

A wide range of disorders produce abdominal pain, and the clinician should be aware of the large number of differential diagnoses. ${ }^{24}$ of great importance is the duration of symptoms-the diagnostic approach to patients with acute abdominal pain is completely different from patients with long-standing abdominal pain. Evaluation should consist of a clinical and psychosocial assessment, observation of symptom-reporting behaviors, a physical examination, and, in the absence of alarm features, conservative efforts to exclude other medical conditions in a costeffective manner. Notably, for patients meeting diagnostic criteria for CAPS who exhibit a longstanding history of pain behaviors, certain psychosocial correlates and no alarm features, the clinical evaluation usually fails to disclose any other specific medical etiology to explain the illness and, in line with this, clinical investigations could be limited. ${ }^{25}$ However, occasionally the evaluation might incidentally identify other medical conditions of uncertain relation to the presentation (eg, hepatic and/or renal cysts or gallstones). Efforts then must be directed toward understanding the relative contributions of CAPS, and the elicited findings or diagnoses, to the pain reported. A number of clinical and behavioral features typify, but are not specific for, CAPS. Their presence may aid in the planning of diagnostic testing and are essential to designing the treatment approach.

\section{Medical History}

Description of the pain. A carefully taken history focused on the description of pain is crucial in these patients, as pain is the central feature. Typically, the pain in CAPS is constant, nearly constant, or frequently recurring, with pain occurring more or less every day. The pain is associated with loss of daily functioning, which is often quite severe (eg, work and school absenteeism, limitations in social activities). The pain is not, or only occasionally, associated with physiological events, such as bowel movements, eating, or menses. In addition, the patient often describes the pain in emotional terms; the pain involves a large anatomic area, rather than a precise location. Patients with CAPS also frequently complain of several other painful extraintestinal symptoms (eg, musculoskeletal pain) and often there is a continuum of painful experiences beginning in childhood or recurring over time. ${ }^{25}$

Symptom behaviors. Although there are symptomrelated behaviors that typify CAPS, they are neither sensitive nor specific and have limited diagnostic value, as they might also occur in patients with a structural disease. These behaviors are usually considered maladaptive but potentially modifiable (Figure 1).

Presence of other medical diagnoses. Symptoms compatible with CAPS may coexist with other structural or functional diagnoses, or at least these diagnoses have been obvious or even dominating the picture initially. This coexistence reflects a transition from more peripherally based afferent neural activity due to bowel dysfunction, to a pattern of central disinhibition usually associated with more constant pain. A proportion of patients with CAPS may also affirm abnormal bowel habit, but without any association with their constant and severe pain. Patients with a structural painful disease might, over time, develop a condition more compatible with CAPS, when the pain pattern evolves as more continuous, severe, and nonresponsive to existing treatment alternatives.

Concurrent psychosocial features and clinical/ psychocial assessment. Patients with CAPS show no consistent psychological profile, but psychosocial difficulties/problems often contribute to poor health outcomes in this group of patients. Many patients with CAPS fulfill diagnostic criteria for comorbid psychiatric diagnoses, including anxiety, depression, and somatization, ${ }^{26}$ but, 
Figure 1. Symptomrelated behaviors are common in CAPS. These symptoms lack sensitivity and specificity to a CAPS diagnosis, but awareness of these behaviors can facilitate the clinical examination.

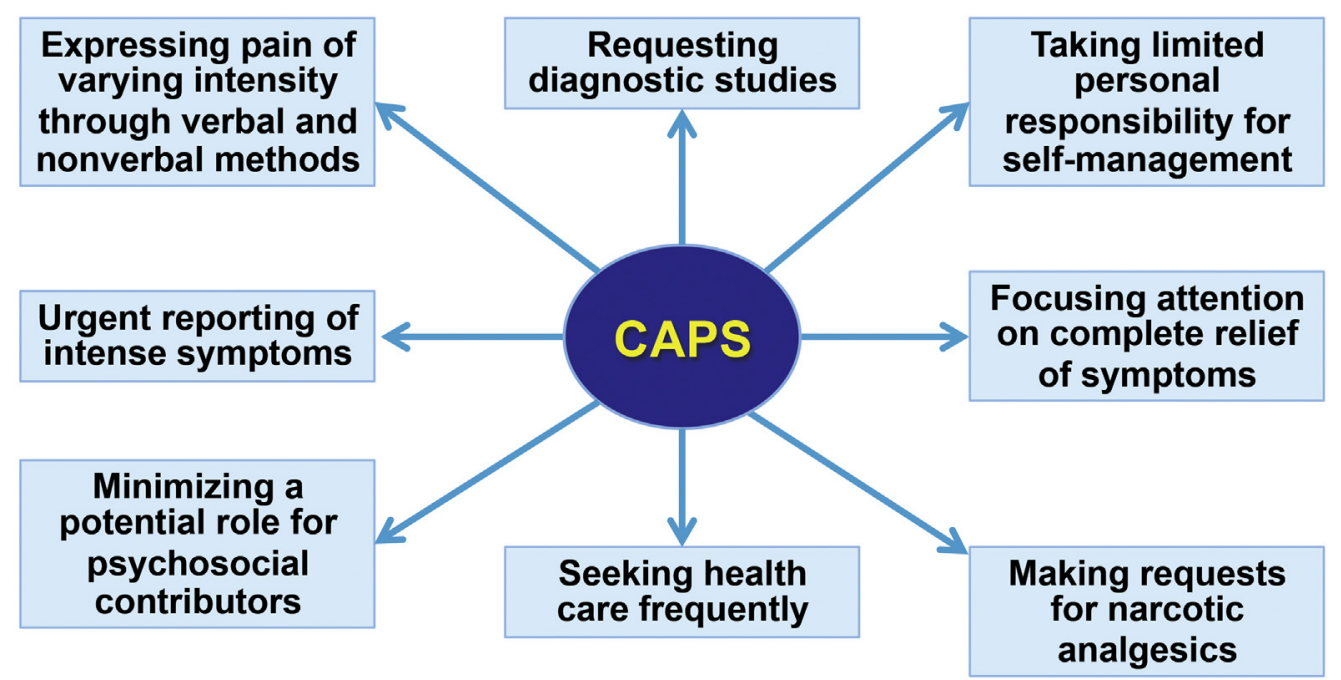

unlike patients with these primary diagnoses, patients with CAPS are often reluctant to accept that these could contribute to their symptom profile. A history of unresolved losses (eg, death of a parent, surgery), ${ }^{27}$ as well as a history of sexual and physical abuse, are common features in patients with CAPS, ${ }^{28}$ but it should be noted that their presence is by no means diagnostic of CAPS, but rather can explain the severity of the condition. Independent of diagnosis, a history of abuse predicts poor health outcomes. ${ }^{29}$ By answering a few questions, the physician can appraise effectively the clinical features of CAPS, identify the key psychosocial contributions to the disorder, and increase confidence in the diagnosis ${ }^{30}$ (Figure 2).

\section{Physical Examination}

The physical examination does not establish a diagnosis of CAPS, and only rarely does it identify other etiologies in chronic pain patients. Nevertheless, there is no substitute for examining the patient to clarify pain location and radiation patterns, and to legitimize the patent's symptoms. Additionally, in the previously uninvestigated patient, important physical findings can direct the diagnostic workup and might expeditiously lead to an underlying cause (eg, abdominal wall pain). ${ }^{24,31}$

Investigations. Tests to exclude other diagnoses should not be done on a routine basis, but based only on the presence of "alarm signs" or "red flags" indicating a clinical suspicion of organic disease. ${ }^{32}$ A minimal diagnostic workup should include routine laboratory tests to exclude inflammation and signs of GI bleeding (anemia, fecal blood loss). If alarm features are identified by history, physical examination or laboratory screening investigations, the physician should examine the patient for causes of abdominal pain other than CAPS. However, in the absence of alarm features or screening abnormalities, no further tests are indicated, and in the presence of longstanding stable symptoms, the

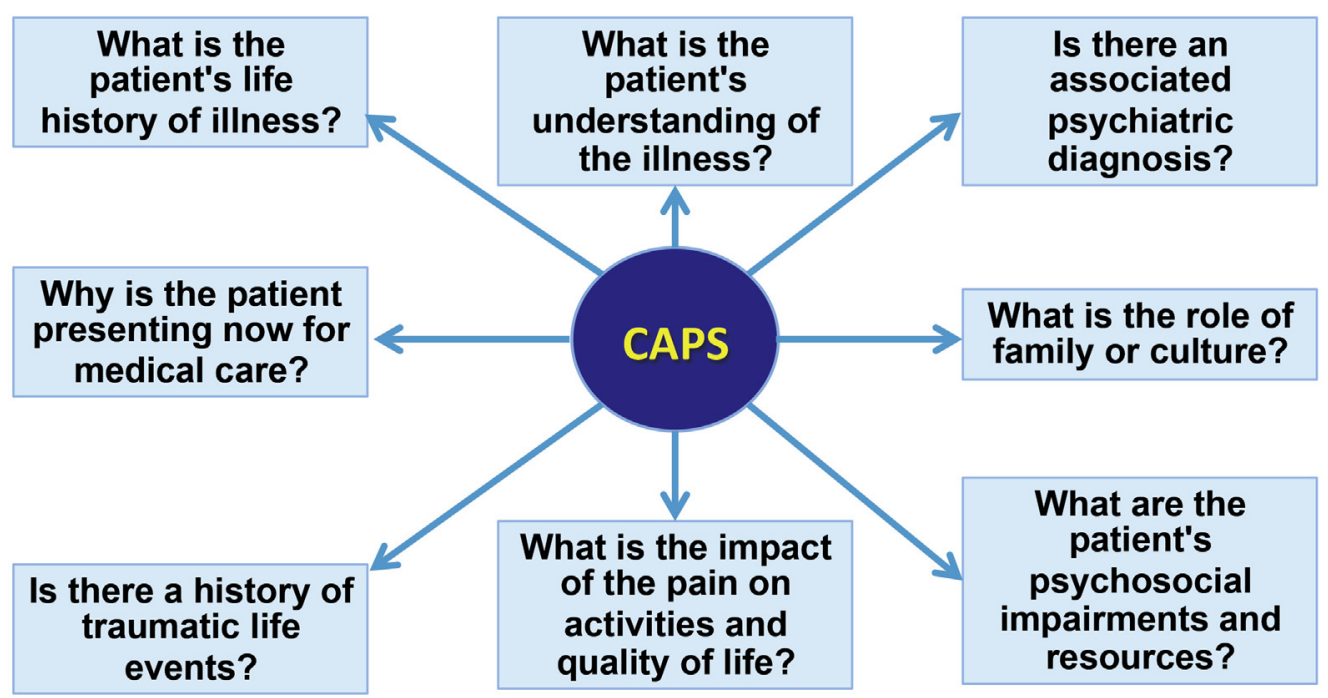

Figure 2. These questions can provide a clinical and psychosocial overview of the patient's condition to support the diagnosis of CAPS and to assist in formulating a treatment plan. 
diagnosis of CAPS is highly probable if all criteria for this diagnosis have been met. $^{33}$ The appropriateness of this approach is supported by several recent studies demonstrating that diagnostic failures are very rare, ${ }^{34}$ and that the health-related quality of life for patients with an FGID does not increase after the patients have undergone investigations. ${ }^{35}$ Figure 3 displays an algorithm for the evaluation of CAPS.

\section{Treatment}

The management of CAPS relies on establishing an effective patient-physician relationship, ${ }^{36}$ following a general treatment plan (eg, setting treatment goals and basing treatment on symptom severity), and offering management that encompasses a combination of treatment options, including pharmacologic and/or psychologic treatments ${ }^{37}$ (Figure 4).

\section{Establishing an Effective Patient-Physician Relationship}

Patients and physicians must share responsibility for the treatment. For example, patients must hold realistic expectations about treatment and the provider can help adjust expectations through questions such as "How do you believe I can be helpful to you?" Patients must be ready to enter into a therapeutic relationship with a provider-in CAPS, the focus needs to move away from evaluation and cure toward facilitating adaptation to constant symptoms. Finally, patients must be ready to take responsibility in their care-this is associated with improvement in clinical outcomes.

Physicians and patients both benefit when physicians listen actively, accept CAPS as a true disorder, offer empathy, use an open-ended question style with matching body language, validate the patients' feelings, ${ }^{38}$ set realistic treatment goals, educate the patient about the nature of their condition, reassure, negotiate and provide choices around treatment rather than directives, maintain boundaries, and are aware of time constraints.

Some additional principles to consider:

1. Base treatment on symptom severity and degree of disability. If pain is continuous and severe, or if the patient is reluctant to participate in a psychological intervention, antidepressants (eg, TCAs or serotoninnorepinephrine reuptake inhibitors [SNRIs]) are used for their analgesic effects.

2. Know when to refer to a mental health professional. Present the psychological referral as a means to help the patient manage the pain and reduce the emotional distress encumbered by the symptoms. Medical care should continue concurrent with psychological treatment.

3. Referral to a multidisciplinary functional GI or pain treatment center. Multidisciplinary functional GI or pain treatment centers provide comprehensive assessment and treatment. Care must be taken to avoid pain centers that focus on opioid treatment, which is contraindicated and raises the risk for NBS.

\section{General Principles of Treatment}

Pharmacologic therapy. Pharmacologic therapy for CAPS can be employed along with the general treatment approaches outlined here. Medical treatment is most effective within the context of a well-developed patientphysician relationship, ${ }^{36}$ and a comprehensive biopsychosocial $^{39}$ treatment plan (Table 1).

Tricyclic antidepressants. TCAs are the most widely used psychotropic agents for treating medical (eg, postherpetic neuralgia, diabetic neuropathy) and functional pain syndromes (eg, fibromyalgia). ${ }^{40-44}$ Their analgesic effect is probably unrelated to the antidepressant effect because these drugs are helpful in many pain syndromes where psychopathology is less prominent or absent, and

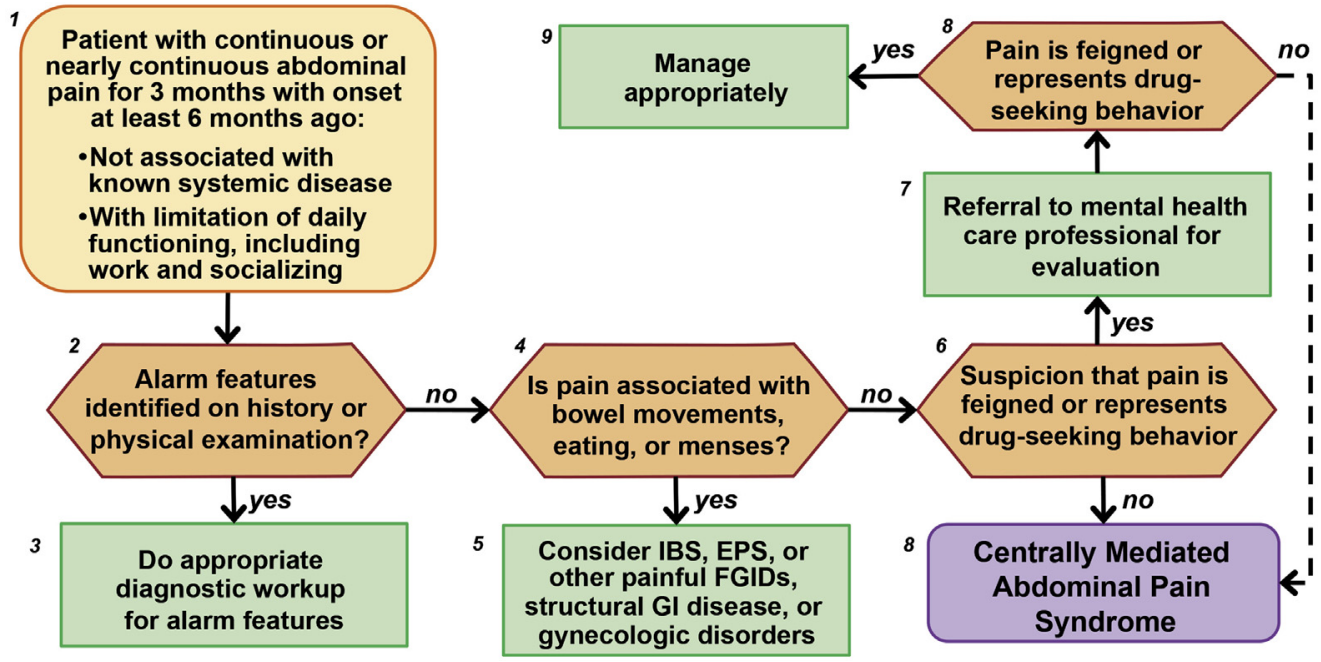

Figure 3. The differential diagnosis for chronic abdominal pain is broad. The evaluation of suspected CAPS can be simplified using this algorithm. (Reprinted from Sperber AD, Drossman DA. Functional abdominal pain syndrome: constant or frequently recurring abdominal pain. Am J Gastroenterol 2010;105:770774). 


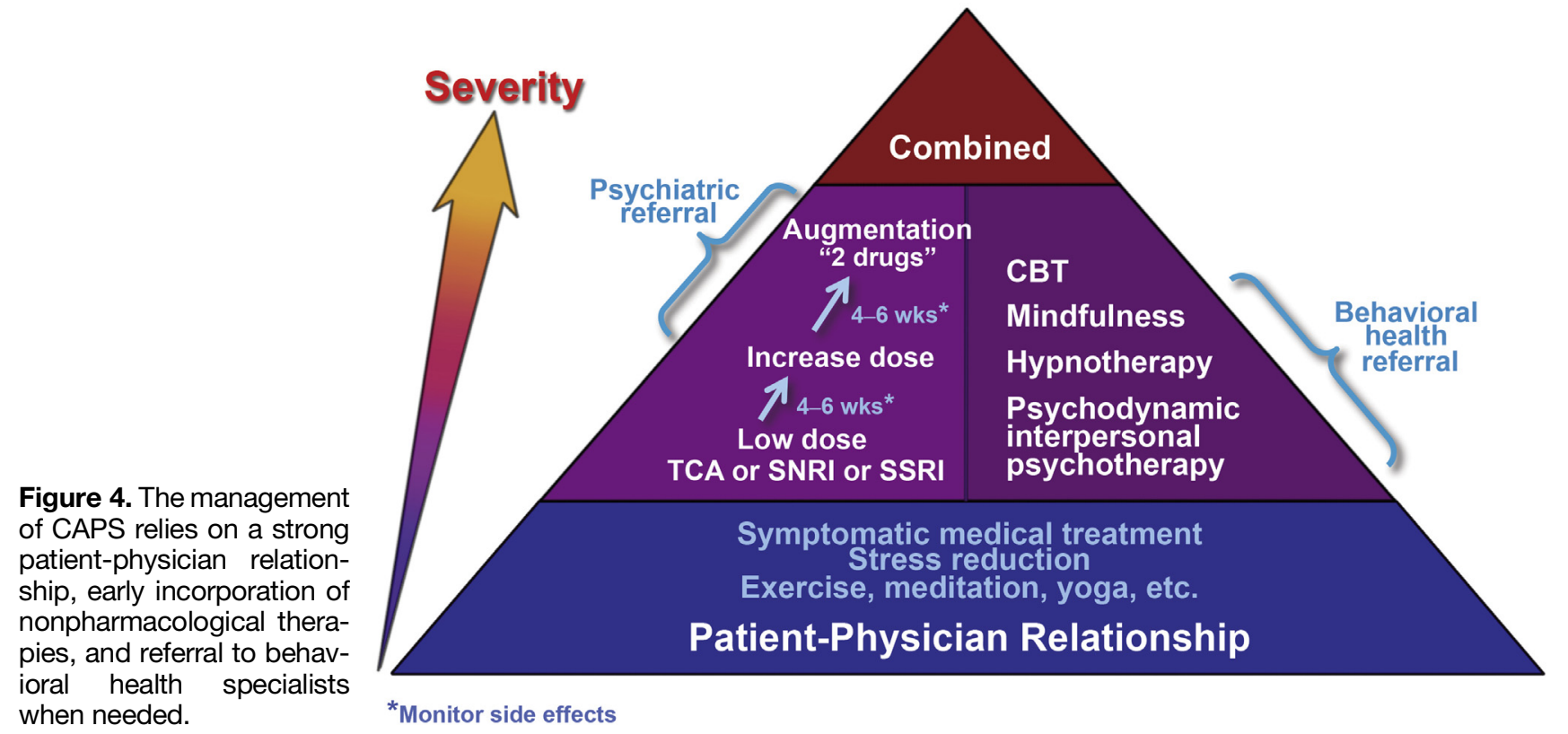

also because they are usually given in low ("subpsychiatric" dosages). Improvement in pain as with desipramine in IBS was not related to blood levels or medication dosage. ${ }^{45}$

TCAs (eg, amitriptyline, imipramine, desipramine, doxepine, and trimipramine) are helpful in relieving pain and reducing IBS symptoms in moderate to severe cases, ${ }^{40,46}$ with a pooled relative risk for clinical improvement with TCA therapy of 1.93. The most common side effects of TCAs include sedation or sometimes agitation, hypotension, constipation, urinary retention, xerostomia, and effects on sleep such as insomnia or nightmares.

Selective serotonin reuptake inhibitors. Selective serotonin reuptake inhibitors (SSRIs) have lesser analgesic effect compared with TCAs, likely due to a lack of effect on noradrenalin synaptic levels, as evidenced by experimental models. ${ }^{47}$ One multicenter study compared amitriptyline (a TCA) to citalopram (an SSRI) and placebo among patients with $\mathrm{FD}^{48}$ and showed significant reduction in symptoms relative to placebo with the TCA, but not the SSRI. SSRIs are probably less potent visceral analgesics than TCAs or SNRIs, but they will have significant effects on global well-being and anxietyspecific GI symptoms. Side effects may include nausea and diarrhea; sexual dysfunction with decreased libido and delayed orgasm; and neurologic/psychiatric symptoms, such as anxiety, nervousness, tremor, insomnia, and nightmares.

\section{Serotonin-norepinephrine reuptake inhib-} itors. The available SNRIs (eg, duloxetine, venlafaxine, desvenlafaxine, and milnacipran), while used for depression, are being used increasingly for treating chronic pain. Their dual effects (analgesic and antidepressant) make them an attractive choice in depressed patients with pain syndromes, ${ }^{49}$ but their benefit for CAPS is theoretical (Table 1).

Decisions relating to which antidepressant to use (or whether to combine them) will depend on several factors, including the agent's potential to address pain and side effects of diarrhea or constipation. These factors depend largely on the main receptor sites of action (Table 2).

Atypical antipsychotics. Quetiapine has been used in lower doses for treating medical patients with anxiety, sleep disturbance, and associated psychological comorbid symptoms, and for augmentation treatment with painful disorders like fibromyalgia. ${ }^{50}$ It can benefit patients with chronic abdominal pain by reducing anxiety, restoring normal sleep patterns, and possibly through a direct analgesic effect. ${ }^{51,52}$ Overall, it appears to augment the

Table 1.Antidepressant treatment for CAPS

\begin{tabular}{|c|c|c|c|}
\hline & Tricyclic antidepressants & $\begin{array}{l}\text { Selective serotonin } \\
\text { reuptake inhibitors }\end{array}$ & $\begin{array}{l}\text { Serotonin-norepinephrine } \\
\text { reuptake inhibitors }\end{array}$ \\
\hline Treatment targets & Pain, depression & $\begin{array}{l}\text { Pain, depression, panic, anxiety, } \\
\text { obsessive compulsive disorder }\end{array}$ & Pain, depression \\
\hline Adverse events & $\begin{array}{l}\text { Sedation, hypo-tension, constipation, } \\
\text { dry mouth/eyes, arrhythmias, } \\
\text { weight gain, sex dysfunction }\end{array}$ & $\begin{array}{l}\text { Insomnia, agitation, diarrhea, } \\
\text { night sweats, headache, } \\
\text { weight loss, sex dysfunction }\end{array}$ & $\begin{array}{l}\text { Nausea, agitation, dizzi-ness, } \\
\text { sleep disturbance, fatigue, } \\
\text { liver dysfunction }\end{array}$ \\
\hline Risk from overdose & Moderate & Low & Minimal \\
\hline Dose adjustment & Yes & Not usual & Not usual \\
\hline
\end{tabular}


Table 2. Antidepressant Receptor Site Effects

\begin{tabular}{|c|c|c|c|c|}
\hline & Norepinephrine & 5-hydroxytryptamine & Histamine & Acetylcholine \\
\hline \multicolumn{5}{|l|}{ TCAs (25-150 mg) } \\
\hline Amitryptaline $\left(3^{\circ}\right)$ & +++ & +++ & ++++ & ++++ \\
\hline Doxepin $\left(3^{\circ}\right)$ & ++ & +++ & ++++ & ++ \\
\hline Desipramine $\left(2^{\circ}\right)$ & +++ & +++ & + & + \\
\hline Nortriptyline $\left(2^{\circ}\right)$ & +++ & + & ++ & ++ \\
\hline \multicolumn{5}{|l|}{ SSRIs (1-2 pills) } \\
\hline Citalopram & nil & ++++ & nil & nil \\
\hline Escitalopram & nil & ++++ & nil & nil \\
\hline Fluoxetine & nil & ++++ & nil & nil \\
\hline Paroxetine & nil & ++++ & nil & nil \\
\hline Sertraline & nil & ++++ & nil & nil \\
\hline \multicolumn{5}{|l|}{ SNRIs (variable) } \\
\hline Venlafaxine & ++ & ++ & nil & nil \\
\hline Duloxetine & +++ & +++ & nil & nil \\
\hline Milnacipran & +++ & ++ & nil & nil \\
\hline
\end{tabular}

benefits of TCAs or SNRIs in patients not having an adequate clinical response.

Miscellaneous psychotropic medications. Mirtazapine, a tetracyclic antidepressant has increased noradrenergic activity and 5-HT1A serotonergic activity, which makes it helpful as an antiemetic and appetite stimulant, leading to weight gain, as in patients with FD. ${ }^{53}$ Buspirone is a nonbenzodiazepine, anti-anxiety agent that can augment the effect of antidepressants, and with 5HT1 agonist effects, ${ }^{54}$ it can improve symptoms of FD. ${ }^{55}$

Anticonvulsants. Anticonvulsants, such as carbamazepine, lamotrigine, and, more recently, the $\alpha 2 \Delta$ ligand agents, gabapentin and pregabalin, have been evaluated in some chronic pain syndromes, but have not been studied for chronic abdominal pain or CAPS. ${ }^{56,57}$ Brain imaging studies showed that pregabalin reduced chronic pain reports and this was associated with a reduction of the usually increased functional connectivity seen between brain regions in chronic pain states. $^{58}$

Analgesics. Most analgesics (eg, aspirin and nonsteroidal anti-inflammatory drugs) offer little benefit because their actions are somatic in location. Narcotic analgesics should be avoided because of the likelihood of addiction and the possibility of narcotic bowel syndrome and other GI side effects. $^{59}$

Augmentation treatment. With CAPS, sequencing high dosages of one medication after another may fail due to incomplete response or side effects. Augmentation involves the use, usually at lower dosages, of 2 or more treatments that act on different receptor sites or areas of the brain to enhance the therapeutic effect. ${ }^{54}$ Augmentation treatment using multiple psychotropic agents should be prescribed in consultation with or by a psychiatrist, psychopharmacologist, or medical physician with advanced training in the use of these medications.

Psychological treatment and antidepressants. An effective augmentation approach is to combine antidepressants with psychological treatment. Antidepressants can improve pain and vegetative signs of depression, while psychological treatments improve higher levels of brain functioning, such as coping, reappraising of maladaptive cognitions, and cognitive adaptation to previous losses and trauma. Psychological treatment can improve adherence to taking a medication, and conversely taking an antidepressant can increase psychic energy to improve the efficiency of the work of therapy. Studies have shown that antidepressants work in subcortical areas, such as the anterior cingulate cortex and insula, to improve connectivity to prefrontal and other cortical areas ("bottom up" effects), and psychological treatments work on prefrontal or cognitive ("executive") areas, "top-down" effects. ${ }^{60}$ The effect size difference for combined treatment can be $50 \%$ or more than either monotherapy treatment. $^{61,62}$ Four classes of psychotherapy hold the most promise in CAPS: cognitive-behavioral therapy, psychodynamic-interpersonal therapy, mindfulness/ acceptance-based therapies and hypnotherapy. These are typically administered individually by a health psychologist or other mental health provider familiar with GI physiology, always in conjunction with medical treatment.

\section{Other Interventions}

Patients seldom gain substantial relief from their symptoms and seek out alternative treatment approaches. However, peripherally based treatments are unlikely to be more effective than centrally targeted modalities.

There is no evidence to support spinal manipulation, and minimal evidence to support transcutaneous electrical nerve stimulation or acupuncture, although the latter may have some putative effect on the opioid system and could be recommended. Neurolytic celiac plexus blockade in benign disease has been restricted to chronic abdominal pain from suspected structural sources, such as chronic pancreatitis, and with only modest success. Many patients with CAPS exhibit erythema ab igne, indicating the excessive use of hot water bottles, electric heating pads, etc, suggesting that heat seems to provide some degree of pain relief despite any direct evidence. Although uncontrolled studies suggest a significant diagnostic and therapeutic benefit of 
laparoscopic adhesiolysis in patients with chronic abdominal pain, ${ }^{63}$ the outcome may be placebo-related, and the detection of unsuspected diagnoses is rare. ${ }^{64}$ Repeated adhesiolysis should be minimized to those situations where it is likely that the adhesions are having a clinical effect such as intermittent small bowel obstruction.

\section{D2. Narcotic Bowel Syndrome/Opiate- Induced Gastrointestinal Hyperalgesia}

\section{Definition}

NBS is characterized by the paradoxical development of, or increases in, abdominal pain associated with continuous or increasing dosages of opioids. ${ }^{59}$ NBS can occur in patients with functional GI disorders or chronic GI diseases (eg, IBD, chronic pancreatitis), with painful malignant or nonmalignant diseases, or even in patients receiving high dosages of narcotics when recovering from surgery. ${ }^{65}$ Patients with NBS will have relief or meaningful improvement of their pain when the opioids are withdrawn. ${ }^{65}$

\section{Epidemiology}

Opioids are the most commonly prescribed drug category in the United States. ${ }^{66}$ Most pain clinicians will not see NBS; its recognition is more familiar in GI practices where patients on opioids are referred for severe abdominal pain and presumed to have an FGID. ${ }^{67}$

D2. Diagnostic Criteria ${ }^{a}$ for Narcotic Bowel Syndrome/ Opioid-Induced Gastrointestinal Hyperalgesia

Must include all of the following:

1. Chronic or frequently recurring abdominal pain $^{b}$ that is treated with acute high-dose or chronic narcotics

2. The nature and intensity of the pain is not explained by a current or previous GI diagnosis ${ }^{c}$

3. Two or more of the following:

a. The pain worsens or incompletely resolves with continued or escalating dosages of narcotics

b. There is marked worsening of pain when the narcotic dose wanes and improvement when narcotics are re-instituted (soar and crash)

c. There is a progression of the frequency, duration, and intensity of pain episodes

${ }^{a}$ Criteria fulfilled for the last 3 months with symptom onset at least 6 months before diagnosis.

${ }^{b}$ Pain must occur most days.

${ }^{c} \mathrm{~A}$ patient may have a structural diagnosis (eg, inflammatory bowel disease, chronic pancreatitis), but the character or activity of the disease process is not sufficient to explain the pain.

\section{Pathophysiology}

Physiological features. While there are several putative mechanisms to explain the central hyperalgesia of opioids, perhaps the most favored is that of glial cell activation in the dorsal horn of the spinal cord, which up-regulates peripheral nociceptive signals going cephalad. ${ }^{68}$ Dorsal horn glia (astrocytes and microglia), when activated, produce proinflammatory cytokines, nitrous oxide, and excitatory amino acids. This leads to central hyperalgesia with enhanced pain. Glial cell activation occurs in response to inflammation or infection, drugs such as morphine, an endogenous chemokines (fractalkine), from peripheral injury, other activated glial cells, or even in response to signals from the central nervous system, which opens the possibility for central effects of stress on peripheral pain facilitation. ${ }^{69}$ The glia cell, the immunocompetent cell of the central nervous system, are activated via Toll-like receptors (TLR4), which modify the pharmacodynamics of opioids by eliciting a proinflammatory reaction with disruption of glutamate homeostasis. ${ }^{70}$ Certain pharmacologic agents could potentially interrupt this paininducing pathway via disruption of TLR4 and TLR2 signaling ${ }^{70}$ including TCAs. ${ }^{71}$

Another potential contributing factor is the bimodal excitatory and inhibitory opioid modulation system in the dorsal horn. The Gi/o protein inhibitory receptor is activated, leading to analgesia with short-term opioid use, but also Gs protein excitatory receptor can be activated to produce hyperalgesia in some individuals when chronic high dosages of opioids are used. ${ }^{72}$ The Gs-coupled excitatory opioid receptors become progressively sensitized during chronic exposure of dorsal root ganglia to opioid agonists over time leading to tolerance of inhibitory pain effects and ultimately hyperalgesia via Gscoupled activation. Clinically, the use of prolonged highdose narcotic agonists may produce opioid hyperalgesia and NBS.

Descending pathways originating from the cingulate and prefrontal cortex, the rostral ventral medulla and periaqueductal gray can produce antinociception, although descending tracts through the dorsolateral funiculus can enhance pro-nociceptive input. ${ }^{73}$ These responses have been demonstrated to occur via activation or inactivation of "on" and "off" cells in the rostral ventral medulla. Activation of the off cells produces an inhibition of nociceptive input, while activation of the on cells is believed to facilitate nociceptive processing within the rostral ventral medulla and descending projections to the spinal cord. ${ }^{74}$ An animal model for NBS has been described in which morphine has led to the development of central and visceral hyperalgesia. ${ }^{75}$ Minocycline, a known inhibitor of microglia activation, resulted in normalization of the hyperalgesia during the morphine treatment.

\section{Clinical Evaluation}

Patients with NBS most often report moderate to severe colicky or constant abdominal pain, which is poorly localized. They may have been prescribed opioids initially for 
intra-abdominal (eg, chronic pancreatitis, inflammatory bowel disease, CAPS) or extra-abdominal (eg, orthopedic pain, fibromyalgia, migraine headaches) conditions, or even develop increasing pain after surgery. Although initially receiving opioids for intermittent pain, patients soon develop tolerance and tachyphylaxis, which require escalating doses for continued clinical benefit. Eventually, reduced or no pain periods diminish and the abdominal pain becomes constant and severe despite ongoing treatment. ${ }^{59}$

The pain may be associated with other GI symptoms consistent with opioid bowel dysfunction, including nausea, vomiting, heartburn, constipation, and either overflow diarrhea or diarrhea from opioid withdrawal. Associated diagnoses may include gastroparesis, pseudo-obstruction, and opioid-induced constipation.

Patients may show psychosocial disturbances, including anxiety, depression, somatization, post-traumatic stress disorder, and personality disorders often associated with high health care use and increased health care expenditures due to procedures, surgery, and medications. ${ }^{65}$ Although these features are not a part of the diagnostic criteria of NBS per se, awareness of them is helpful in treatment planning.

Patients may have had extensive laboratory studies done, which are usually normal, and radiologic studies might show colonic fecal retention. Cross-sectional imaging has usually been done to exclude obstruction, pancreatitis, inflammatory or ischemic bowel, or other intra-abdominal pathology. These negative studies, in addition to a focused history and physical examination and meeting the diagnostic criteria, should be adequate to make a diagnosis of NBS.

NBS is a positive diagnosis that can occur solely (eg, in a patient developing abdominal pain after an operation or treatment for back pain), or it can be present alongside other structural GI diseases, when the pain is out of proportion to the pain inferred to be from the structural disease. For example, patients may have had pancreatitis with resolution of the lipase but worsening pain from the NBS, or may have inflammatory bowel disease without complications of bowel obstruction, deep ulcers, or serologic evidence for inflammation. The diagnosis is particularly challenging when the patient has active bowel disease and is also on opioids in the clinical setting of worsening abdominal pain. As such, there is no specific diagnostic evaluation recommended other than the good clinical judgment required to evaluate the activity of other comorbid medical diseases. In these cases, it might be reasonable to detoxify patients as a therapeutic trial to see if NBS is present.

There is no particular time frame or dosage of opioids required for diagnosis because NBS can occur within a few weeks and with varying dosages. Therefore, the diagnosis is based on the development of the clinical features in a setting of opioid use, and it is observed that patients most often are taking, on average, $75 \mathrm{mg}$ or more daily of oral morphine equivalent. ${ }^{65}$

A subset of patients with abdominal pain on opioids may have opioid use disorder. ${ }^{76}$ These patients take opioids in larger amounts than intended, with a persistent desire to continue and crave for them, and fail to fulfill normal work, school, or home obligations. ${ }^{77}$ Patients with these features should be referred to a substance abuse program, as the likelihood of successful management of these patients in a medical setting is extremely low.

\section{Treatment}

Understanding the patient. It helps to understand that most patients with NBS want to be treated, but might not see reduction of opioids as a logical option. These patients believe that opioids have been "all that has helped" and fear being abandoned with worsening pain. They also feel stigmatized by others who they perceive see them as "drug seeking" or having a psychiatric problem.

Clinician considerations in the treatment. A sound patient-physician relationship through good communication skills is a prerequisite to the treatment of patients with NBS. ${ }^{78}$ The clinician must feel committed to work with these patients and be aware of possible negative feelings toward patients that $\mathrm{s} /$ he perceives as "difficult."

Educating about the treatment. Once a commitment is made to treat, the physician must engage with the patient and discuss NBS and options for treatment, including opiate detoxification. The Current Opioid Misuse Measure ${ }^{79}$ is a useful tool to determine the severity of misuse and likelihood of detoxification. The treatment protocol discussed here involves complete detoxification because there is only evidence of clinical improvement with this approach ${ }^{65}$ and no evidence that NBS can be treated with continuation of opioids.

Negotiating the treatment requires mutual trust and patient engagement toward a shared plan of care, ${ }^{59}$ which can be facilitated through empathy, acceptance, and validation of the reality of the pain and its impact on the patient's life, an open dialogue about the mechanism of NBS and the rationale for the recommended treatment, including the specifics of opioid detoxification and eliciting/addressing the patient's concerns directly. If the patient says opioids have been the only effective treatment, the clinician can note that, despite using high-dose opioids, the patient is still not achieving benefit or it is incomplete; describe the value of more effective treatments for the pain (eg, tricyclic or SNRI antidepressants and central anxiety-reducing agents) to use during and after detoxification; note that once beginning the program, the protocol for opiate reduction will not change, but alternative agents can be used for pain and anxiety; provide realistic goals from the detoxification, which are to improve the pain (not necessarily achieve complete pain resolution) over the course of months; enlist friends and family members in discussions of the goals and treatment to ensure their support during the process and also to help prevent relapse; indicate the role of ancillary providers (eg, psychologists or psychiatrist, primary care physician, and physician assistant) to help in the process; and reaffirm their willingness to continue with the patient in the care regardless of the outcome.

Treatment plan is provided in Table 2 .

Clinical outcome. The single outcome study in NBS detoxification included 39 patients who were systematically detoxified in an inpatient facility during a 7-day period with 
a success rate of $89.7 \%$. Sixty percent were considered "responders"; 11\% percent had worse pain after detoxification. ${ }^{65}$ This committee believes that inpatient programs provide much more control over detoxification, and can usually be done in 1 week rather than outpatient programs, which take several weeks.

\section{Conclusions}

We described a set of GI pain disorders with a central determinant. These disorders are increasingly common and complex and must be approached from a comprehensive biopsychosocial model. The doctor-patient relationship is at the core of all evaluation and management decisions. CAPS is distinguished by chronic, unrelenting pain that interferes with several life domains and has no clear triggers, such as bowel movements, food, or menses. It may be at the far end of the spectrum of other FGIDs, such as IBS. Our newest disorder, NBS/opioid-induced GI hyperalgesia is characterized by the paradoxical development of worsening abdominal pain associated with increased use of opioids. The only treatment for NBS is to withdraw opioids, which requires a thoughtful and collaborative approach. Both CAPS and NBS require a substantial research effort over the next several years to more fully charaterize their prevalence and features, understand their unique pathophysiology and identify more effective therapeutic targets.

\section{References}

1. Longstreth GF, Yao JF. Irritable bowel syndrome and surgery: a multivariable analysis. Gastroenterology 2004; 126:1665-1673.

2. Reimerink JJ, van der Laan MJ, Koelemay MJ, et al. Systematic review and meta-analysis of populationbased mortality from ruptured abdominal aortic aneurysm. Br J Surg 2013;100:1405-1413.

3. Clouse RE, Mayer EA, Aziz Q, et al. Functional abdominal pain syndrome. Gastroenterology 2006;130:1492-1497.

4. Drossman DA, Li Z, Andruzzi E, et al. US householder survey of functional gastrointestinal disorders. Prevalence, sociodemography, and health impact. Dig Dis Sci 1993;38:1569-1580.

5. Thompson WG, Irvine EJ, Pare $P$, et al. Functional gastrointestinal disorders in Canada: first populationbased survey using Rome II criteria with suggestions for improving the questionnaire. Dig Dis Sci 2002;47:225-235.

6. Bharucha AE, Camilleri M. Functional abdominal pain in the elderly. Gastroenterol Clin North Am 2001; 30:517-529.

7. Koloski NA, Talley NJ, Boyce PM. Epidemiology and health care seeking in the functional Gl disorders: a population-based study. Am J Gastroenterol 2002; 97:2290-2299.

8. Maxton DG, Whorwell PJ. Use of medical resorce and attitudes to health care of patients with chronic abdominal pain. Br J Med Econ 1992;2:75-79.

9. Van Oudenhove L, Vandenberghe J, Dupont P, et al. Abnormal regional brain activity during rest and (anticipated) gastric distension in functional dyspepsia and the role of anxiety: a H(2)(15)O-PET study. Am J Gastroenterol 2010;105:913-924.

10. Devor M. Neuropathic pain: what do we do with all these theories? Acta Anaesthesiol Scand 2001;45:1121-1127.

11. Sperber AD, Morris CB, Greemberg L, et al. Development of abdominal pain and IBS following gynecological surgery: a prospective, controlled study. Gastroenterology 2008;134:75-84.

12. Jiang Z, Dinov ID, Labus J, et al. Sex-related differences of cortical thickness in patients with chronic abdominal pain. PLoS One 2013;8:e73932.

13. Labus JS, Dinov ID, Jiang Z, et al. Irritable bowel syndrome in female patients is associated with alterations in structural brain networks. Pain 2014;155:137-149.

14. Zeng F, Qin W, Yang Y, et al. Regional brain structural abnormality in meal-related functional dyspepsia patients: a voxel-based morphometry study. PLoS One 2013;8:e68383.

15. Mayer EA, Collins SM. Evolving pathophysiologic models of functional gastrointestinal disorders. Gastroenterology 2002;122:2032-2048.

16. Van Oudenhove L, Vandenberghe J, Dupont P, et al. Regional brain activity in functional dyspepsia: a H(2)(15) O-PET study on the role of gastric sensitivity and abuse history. Gastroenterology 2010;139:36-47.

17. Wegener ST, Castillo RC, Haythornthwaite J, et al. Psychological distress mediates the effect of pain on function. Pain 2011;152:1349-1357.

18. Drossman DA. Abuse, trauma, and Gl illness: is there a link? Am J Gastroenterol 2011;106:14-25.

19. Leung L. Pain catastrophizing: an updated review. Indian J Psychol Med 2012;34:204-217.

20. Esteve R, Ramirez-Maestre C. Pain fear avoidance and pain acceptance: a cross-sectional study comparing their influence on adjustment to chronic pain across three samples of patients. Ann Behav Med 2013;46:169-180.

21. Saunders D. Coping with chronic pain: what can we learn from pain self-efficacy beliefs? J Rheumatol 2004; 31:1032-1034.

22. Muller L, Korsgaard H, Ethelberg S. Burden of acute gastrointestinal illness in Denmark 2009: a population-based telephone survey. Epidemiol Infect 2012;140:290-298.

23. McCracken LM, Eccleston C. Coping or acceptance: what to do about chronic pain? Pain 2003;105:197-204.

24. Yamada T, Inadomi JM, Bhattacharya R, et al. Approach to the patient with abdominal pain. In: Yamada $\mathrm{T}$, ed. Yamada's Handbook of Gastroenterology. 3rd ed. Philadelphia: Wiley-Blackwell, 2013:58-69.

25. Drossman DA. Functional abdominal pain syndrome. Clin Gastroenterol Hepatol 2004;2:353-365.

26. Drossman DA. Diagnosing and treating patients with refractory functional gastrointestinal disorders. Ann Intern Med 1995;123:688-697.

27. Drossman DA. Patients with psychogenic abdominal pain: six years' observation in the medical setting. Am J Psychiatry 1982;139:1549-1557.

28. Leserman J, Drossman DA, Li Z, et al. Sexual and physical abuse history in gastroenterology practice: how 
types of abuse impact health status. Psychosom Med 1996;58:4-15.

29. Drossman DA, Leserman J, Hu JB. Gastrointestinal diagnosis, abuse history, and effects on health status. Gastroenterology 1996;111:1159-1161.

30. Grover M, Drossman DA. Functional abdominal pain. Curr Gastroenterol Rep 2010;12:391-398.

31. Khan S, Chang L. Diagnosis and management of IBS. Nat Rev Gastroenterol Hepatol 2010;7:565-581.

32. Vanner SJ, Depew WT, Paterson WG, et al. Predictive value of the Rome criteria for diagnosing the irritable bowel syndrome. Am J Gastroenterol 1999; 94:2912-2917.

33. Sperber AD, Drossman DA. Functional abdominal pain syndrome: Constant or frequently recurring abdominal pain. Am J Gastoenterol 2010;105:770-774.

34. Chey WD, Nojkov B, Rubenstein JH, et al. The yield of colonoscopy in patients with non-constipated irritable bowel syndrome: results from a prospective, controlled US trial. Am J Gastroenterol 2010;105: 859-865.

35. Spiegel BM, Gralnek IM, Bolus R, et al. Is a negative colonoscopy associated with reassurance or improved health-related quality of life in irritable bowel syndrome? Gastrointest Endosc 2005;62:892-899.

36. Drossman DA. 2012 David Sun Lecture: helping your patient by helping yourself: How to improve the patientphysician relationship by optimizing communication skills. Am J Gastroenterol 2013:521-528.

37. Sperber AD, Drossman DA. Review article: the functional abdominal pain syndrome. Aliment Pharmacol Ther 2011;33:514-524.

38. Roter DL, Hall JA. Doctors Talking With Patients/Patients Talking With Doctors: Improving Communication in Medical Visits. Westport, CT: Greenwood Publishing Group, 1992.

39. Drossman DA. Presidential address: gastrointestinal illness and biopsychosocial model. Psychosom Med 1998;60:258-267.

40. Ford AC, Talley NJ, Schoenfeld PS, et al. Efficacy of antidepressants and psychological therapies in irritable bowel syndrome: systematic review and meta-analysis. Gut 2009;58:367-378.

41. Fishbain DA, Cutler RB, Rosomoff HL, et al. Do antidepressants have an analgesic effect in psychogenic pain and somatoform pain disorder? A meta-analysis. Psychosom.Med 1998;60:503-509.

42. Drossman DA, Toner BB, Whitehead WE, et al. Cognitivebehavioral therapy versus education and desipramine versus placebo for moderate to severe functional bowel disorders. Gastroenterology 2003;125:19-31.

43. Drossman DA. Beyond tricyclics: new ideas for treating patients with painful and refractory functional Gl symptoms. Am J Gastroenterol 2009;104:2897-2902.

44. Mic JA, Ardid D, Berrocoso E, et al. Antidepressants and pain. Trends Pharmacol Sci 2006;27:348-354.

45. Halpert A, Dalton CB, Diamant NE, et al. Clinical response to tricyclic antidepressants in functional bowel disorders is not related to dosage. Am J Gastroenterol 2005;100:664-671.
46. Ruepert L, Quartero AO, de Wit NJ, et al. Bulking agents, antispasmodics and antidepressants for the treatment of irritable bowel syndrome. Cochrane Database Syst Rev 2011:CD003460.

47. Bomholt SF, Mikkelsen JD, Blackburn-Munro G. Antinociceptive effects of the antidepressants amitriptyline, duloxetine, mirtazapine and citalopram in animal models of acute, persistent and neuropathic pain. Neuropharmacology 2005;48:252-263.

48. Locke GR, Bouras EP, Howden CW, et al. The Functional Dyspepsia Treatment Trial (FDTT) key results. Gastroenterology 2013;144:S140.

49. Gaynor PJ, Gopal M, Zheng W, et al. Duloxetine versus placebo in the treatment of major depressive disorder and associated painful physical symptoms: a replication study. Curr Med Res Opin 2011;27:1859-1867.

50. Potvin S, Morin M, Cloutier C, et al. Add-on treatment of quetiapine for fibromyalgia: a pilot, randomized, doubleblind, placebo-controlled 12-week trial. J Clin Psychopharmacol 2012;32:684-687.

51. Fishbain DA, Cutler RB, Lewis J, et al. Do the secondgeneration "atypical neuroleptics" have analgesic properties? A structured evidence-based review. Pain Med 2004;5:359-365.

52. Grover M, Dorn SD, Weinland SR, et al. Atypical antipsychotic Quetiapine in the management of severe, refractory functional gastrointestinal disorders. Dig Dis Sci 2009;54:1284-1291.

53. Aderka IM, McLean CP, Huppert JD, et al. Fear, avoidance and physiological symptoms during cognitivebehavioral therapy for social anxiety disorder. Behav Res Ther 2013;51:352-358.

54. Trivedi MH, Fava M, Wisniewski SR, et al. Medication augmentation after the failure of SSRIs for depression. N Engl J Med 2006;354:1243-1252.

55. Rosoff H, John RS, Prager F. Flu, risks, and videotape: escalating fear and avoidance. Risk Anal 2012; 32:729-743.

56. Gale JD, Houghton LA. Alpha 2 Delta (alpha(2)delta) ligands, gabapentin and pregabalin: what is the evidence for potential use of these ligands in irritable bowel syndrome. Front Pharmacol 2011;2:28.

57. Houghton LA, Fell C, Whorwell PJ, et al. Effect of a second-generation alpha2delta ligand (pregabalin) on visceral sensation in hypersensitive patients with irritable bowel syndrome. Gut 2007;56:1218-1225.

58. Harris RE, Napadow V, Huggins JP, et al. Pregabalin rectifies aberrant brain chemistry, connectivity, and functional response in chronic pain patients. Anesthesiol 2013;119:1453-1464.

59. Grunkemeier DMS, Cassara JE, Dalton CB, et al. The narcotic bowel syndrome: clinical features, pathophysiology, and management. Clin Gastroenterol Hepatol 2007:5:1126-1139.

60. Goldapple K, Segal Z, Garson C, et al. Modulation of cortical-limbic pathways in major depression: treatmentspecific effects of cognitive behavior therapy. Arch Gen Psychiatry 2004;61:34-41.

61. Keller MB, McCullough JP, Klein DN, et al. A comparison of nefazodone, the cognitive behavioral-analysis system 
of psychotherapy, and their combination for the treatment of chronic depression. N Engl J Med 2000; 342:1462-1470.

62. Holroyd KA, O'Donnell FJ, Stensland J, et al. Management of chronic tension-type headache with tricyclic antidepressant medication, stress management therapy, and their combination. JAMA 2001;285: 2208-2215.

63. Onders RP, Mittendorf EA. Utility of laparoscopy in chronic abdominal pain. Surgery 2003;134:549-552; discussion 552-554.

64. Di Lorenzo N, Coscarella G, Lirosi F, et al. [Impact of laparoscopic surgery in the treatment of chronic abdominal pain syndrome]. Chir Ital 2002; 54:367-378.

65. Drossman DA, Morris CB, Wrennall CE, et al. Diagnosis, characterization, and 3-month outcome after detoxification of 39 patients with narcotic bowel syndrome. Am J Gastroenterol 2012;107:1426-1440.

66. Trescot AM, Boswell MV, Atluri SL, et al. Opioid guidelines in the management of chronic non-cancer pain. Pain Physician 2006;9:1-39.

67. Farmer AD, Ferdinand E, Aziz Q. Opioids and the gastrointestinal tract-a case of narcotic bowel syndrome and literature review. J Neurogastroenterol Motil 2013;19:94-98.

68. Watkins LR, Milligan ED, Maier SF. Glial activation: a driving force for pathological pain. Trends Neurosci $2001 ; 24: 450-455$.

69. Watkins LR, Hutchinson MR, Johnston IN, et al. Glia: novel counter-regulators of opioid analgesia. Trends Neurosci 2005;28:661-669.

70. Hutchinson MR, Shavit Y, Grace PM, et al. Exploring the neuroimmunopharmacology of opioids: an integrative review of mechanisms of central immune signaling and their implications for opioid analgesia. Pharmacol Rev 2011;63:772-810.
71. Hutchinson MR, Loram LC, Zhang Y, et al. Evidence that tricyclic small molecules may possess toll-like receptor and myeloid differentiation protein 2 activity. Neuroscience 2010;168:551-563.

72. Crain SM, Shen KF. Antagonists of excitatory opioid receptor functions enhance morphine's analgesic potency and attenuate opioid tolerance/dependence liability. Pain 2000;84:121-131.

73. Porreca F, Ossipov MH, Gebhart GF. Chronic pain and medullary descending facilitation. Trends Neurosci 2002; 25:319-325.

74. Fields HL. Is there a facilitating component to central pain modulation? Am Pain Soc J 1992;1:71-78.

75. Agostini S, Eutamene H, Cartier C, et al. Evidence of central and peripheral sensitization in a rat model of narcotic bowel like-syndrome. Gastroenterology 2010; 139:553-563.

76. Brabant M, Brissette S, Lauzon P, et al. [Opioid use disorder in patients with chronic non-cancer pain]. Sante Ment Que 2014;39:117-132.

77. Hasin DS, O’Brien CP, Auriacombe M, et al. DSM-5 criteria for substance use disorders: recommendations and rationale. Am J Psychiatry 2013;170:834-851.

78. Dekel R, Drossman DA, Sperber AD. The use of psychotropic drugs in irritable bowel syndrome. Expert Opin Investig Drugs 2013;22:329-339.

79. Butler SF, Budman SH, Fernandez KC, et al. Development and validation of the Current Opioid Misuse Measure. Pain 2007;130:144-156.

Reprint requests

Address requests for reprints to: Laurie Keefer, PhD, Division of Gastroenterology, Icahn School of Medicine at Mount Sinai, 17 East 102nd Street, 5th Floor, New York, NY 10029. e-mail: laurie.keefer@mssm.edu; fax: (646) 537-8921.

Conflicts of interest

The authors disclose no conflicts. 